\title{
Transcriptional Profiles of Drought-Related Genes in Modulating Metabolic Processes and Antioxidant Defenses in Lolium multiflorum
}

\author{
Ling Pan ${ }^{1}$, Xinquan Zhang ${ }^{1 *}$, Jianping Wang ${ }^{2}$, Xiao Ma ${ }^{1}$, Meiliang Zhou ${ }^{3}$, LinKai Huang ${ }^{1}$, \\ Gang Nie ${ }^{1}$, Pengxi Wang ${ }^{1}$, Zhongfu Yang ${ }^{1}$ and $\mathrm{Ji} \mathrm{Li}^{1}$ \\ ${ }^{1}$ Department of Grassland Science, Sichuan Agricultural University, Chengdu, China, ${ }^{2}$ Agronomy Department, University of \\ Florida, Gainesville, FL, USA, ${ }^{3}$ Department of Crop Molecular Breeding, Biotechnology Research Institute, Chinese Academy \\ of Agricultural Sciences, Beijing, China
}

OPEN ACCESS

Edited by:

Mohammad Anwar Hossain, Bangladesh Agricultural University,

Bangladesh

Reviewed by:

Bin Xu,

Naniing Agricultural University, China

YiXu,

Rutgers University, USA

*Correspondence:

Xinquan Zhang

zhangxq@sicau.edu.cn

Specialty section:

This article was submitted to

Plant Biotechnology,

a section of the journal

Frontiers in Plant Science

Received: 03 November 2015

Accepted: 01 April 2016

Published: 25 April 2016

Citation:

Pan L, Zhang X, Wang J, Ma X, Zhou M, Huang L, Nie G, Wang $P$, Yang $Z$ and Li J (2016) Transcriptional Profiles of Drought-Related Genes in Modulating Metabolic Processes and

Antioxidant Defenses in Lolium multiflorum. Front. Plant Sci. 7:519.

doi: 10.3389/fpls.2016.00519
Drought is a major environmental stress that limits growth and development of cool-season annual grasses. Drought transcriptional profiles of resistant and susceptible lines were studied to understand the molecular mechanisms of drought tolerance in annual ryegrass (Lolium multiflorum L.). A total of 4718 genes exhibited significantly differential expression in two L. multiflorum lines. Additionally, up-regulated genes associated with drought response in the resistant lines were compared with susceptible lines. Gene ontology enrichment and pathway analyses revealed that genes partially encoding drought-responsive proteins as key regulators were significantly involved in carbon metabolism, lipid metabolism, and signal transduction. Comparable gene expression was used to identify the genes that contribute to the high drought tolerance in resistant lines of annual ryegrass. Moreover, we proposed the hypothesis that short-term drought have a beneficial effect on oxidation stress, which may be ascribed to a direct effect on the drought tolerance of annual ryegrass. Evidence suggests that some of the genes encoding antioxidants (HPTs, GGT, AP, 6-PGD, and G6PDH) function as antioxidant in lipid metabolism and signal transduction pathways, which have indispensable and promoting roles in drought resistance. This study provides the first transcriptome data on the induction of drought-related gene expression in annual ryegrass, especially via modulation of metabolic homeostasis, signal transduction, and antioxidant defenses to improve drought tolerance response to short-term drought stress.

Keywords: Lolium multiflorum L, differentially expressed genes, drought tolerance, metabolic processes, antioxidant defense

\section{INTRODUCTION}

Water availability plays a significant role in the transportation of metabolites and enzymatic reactions in plants, and also plays roles in the hydrolytic breakdown of proteins, lipids, and carbohydrates (Bewley and Black, 1994; Białecka and Kêpczyñski, 2010). Previous studies (Mittler, 2006; Liu et al., 2015) have reported that drought tolerance in plants is ascribed to the comprehensive function of multiple pathways. For example, there is a general increase in metabolite 
levels under drought stress, including amino acids, sugars, and alcohols (Witt et al., 2012). Similarly, drought tolerant plants have reaction mechanisms to maintain cellular homeostasis by lipid metabolism and can regulate metabolic homeostasis (Da Silva et al., 1974). The metabolite changes contribute to the number of drought-responsive genes when the plant is under abiotic stress. The genes that encode structural proteins and regulatory proteins involved in metabolic pathways have been shown to be strongly associated with drought tolerance (Song et al., 2005; Xiao et al., 2006). Actually, a number of genes are known to be related to early response to stress, and are thought to be involved in metabolic processes possessing adaptation capacity to abiotic stress (Puranik et al., 2011). The identification such genes has been suggested as representing a promising approach toward the improvement of drought tolerance in crops (Munns, 2005). Responsive genes have been identified through high-throughput sequencing technology, which is a powerful method that is used to analyze changes in cell morphology, gene expression, and physiological and biochemical metabolism for plants under abiotic and biotic stress (Lan et al., 2012).

Drought stress will inevitably result in oxidative damage in plants due to over production of reactive oxygen species (ROS), which are highly reactive and toxic. High ROS level not only cause damage to proteins, lipids, and carbohydrates, but also acts as signal molecules (Petrov et al., 2015). It was recently reported that elevated antioxidant levels are associated with plant tolerance to abiotic stressors (Sudhakar et al., 2001). For example, Mn-SOD, an important antioxidant enzyme, may play a role in the drought tolerance of rice (Wang et al., 2005). The overexpression of ascorbate peroxidase (APX) in Nicotiana tabacum chloroplasts was shown to enhance salt and drought tolerance in the species (Badawi et al., 2004a). Similarly, the overexpression of monodehydroascorbate reductase (MDAR) in transgenic tobacco can increase salt tolerance (Badawi et al., 2004b). Additionally, the overexpression of glutathione S-transferases (GST) and dehydroascorbate reductase (DHAR) enhances plant tolerance to various abiotic stressors (Eltayeb et al., 2007). These results suggest that genes encoding antioxidant enzymes are essential for improving drought tolerance across several plant genera. Therefore, to study antioxidant systems has an important meaning on displaying the mechanism of stress resistance for the plants subjected to abiotic stress.

Drought is a common environmental stress on annual grass productivity in low rainfall areas around the world. The decrease of annual precipitation has become the most critical factor that effects annual ryegrass (Lolium multiflorum L.) germination and establishment (Wang et al., 2005). Annual ryegrass is an important short-duration, cool-season grass that is widely distributed among the world. In southern China, it is most widely used as an annual forage crop and has an increasing area for forage production (Zhang et al., 2008). Although annual ryegrass is a drought resistant grass and has a high root growth capacity, drought still causes a dramatic reduction of tillers and leaves (Antolin et al., 1995).

There is little information concerning the identification of drought-related genes in annual ryegrass. In this study we explored antioxidant-related genes that may contribute to drought tolerance in annual ryegrass. Here we report the results of the regulatory mechanism of annual ryegrass in response to short-term drought stress. The identification of droughtrelated genes has a vital role in the modulation of metabolic processes and development of antioxidant defenses to improve drought tolerance of annual ryegrass. This study aimed to provide candidate genes to facilitate the genetic improvement of annual ryegrass for its use as a sustainable forage crop.

\section{MATERIALS AND METHODS \\ Plant Materials and Drought Stress Treatments}

Seeds of two L. multiflorum lines, "Abundant 10 (droughtresistant)" and "Adrenalin 11 (drought susceptible)," were germinated at $25^{\circ} \mathrm{C}$ on filter paper that was wetted with distilled water. After seven days of growth, seedlings were transplanted into plastic pots $(5 \times 5 \mathrm{~cm})$ filled with the Hoagland's nutrient solution in temperature-controlled growth chambers. The environmental conditions were as follows: temperature $25^{\circ} \mathrm{C}$ during the day and $18^{\circ} \mathrm{C}$ at night, photon flux density 900 umolm $^{-2} \mathrm{~s}^{-1}$, a photoperiod of $16 / 8 \mathrm{~h}$ for the day/night cycle, and a relative humidity of $60 \%$.

To study the effects of short-term drought stress on the physiological response of two L. multiflorum lines, the 20day-old seedlings were split into two halves. One half of the seedlings were used as the control, and the other half of seedlings were naturally air dried (drought treatment) for 1 and $2 \mathrm{~h}$, respectively. The drought-stressed and well-watered seedlings were immediately frozen in liquid nitrogen for RNA-Seq. The leaf transpiration rate was determined by a gravimetric method (Aroca et al., 2003). Leaf water potential was calculated with the formula described by Boyer (1970). Hydrogen peroxide content $\left(\mathrm{H}_{2} \mathrm{O}_{2}\right)$, Malondialdehyde (MDA) concentration, superoxide dismutase (SOD), catalase (CAT), dehydroascorbate reductase (DHAR), and monodehydroasorbate reductase (MDHAR) activities, glucose and fructose concentrations were assayed separately with hydrogen peroxide, MDA, SOD, CAT, DHAP, MDHAR, glucose, and fructose assay kits (Comin Biotechnology Co., Ltd. Suzhou, China). Statistical analysis was performed by one-way ANOVA using SPSS Statistics 20.0. Least Significant Difference (LSD) values were calculated when specific parameters changed significantly $(P<0.05)$.

\section{RNA Extraction and Library Preparation}

Ten individual plants were pooled to create one treatment, and two biological replicates were used for all RNA-Seq analysis for each treatment. Total RNA of each sample was extracted using the Trizol reagent (Agilent RNA 6000 nano Reagents) according to the manufacturer's instructions. The total RNA concentration, RNA integrity number (RIN), and 28S/18S were detected using an Agilent 2100 Bioanalyer (Agilent RNA 6000 Nano Kit). The purity of the samples was tested using a NanoDrop. After RNA isolation and quality assessment, samples were stored at $-80^{\circ} \mathrm{C}$ until the cDNA library construction and transcriptomic assay were completed. Only those samples with an RIN between 6 and 
7 and a $28 \mathrm{~S} / 18 \mathrm{~S}$ ratio within the range of $1.5-2$ were qualified for cDNA library preparation.

\section{Library Construction and Sequencing}

A total of 5 ug of total RNA per sample was used to construct the cDNA library using the NEB Next Ultra RNA Library Prep Kit for Illumina (New England Biol-abs (NEB), USA). Initially, the total RNA sample was digested using Dnase I (NEB) and purified by oligo-dT beads (Dynabeads mRNA purification kit, Invitrogen). Following purification, the poly (A)-containing mRNA was fragmented into 200-250 bp pieces using fragment buffer (Ambion), and the first-strand cDNA was synthesized using the N6 primer, First Strand Master Mix and Super Script II reverse transcription (Invitrogen). The reaction conditions were as follows: $25^{\circ} \mathrm{C}$ for $10 \mathrm{~min} ; 42^{\circ} \mathrm{C}$ for $30 \mathrm{~min} ; 70^{\circ} \mathrm{C}$ for $15 \mathrm{~min} ; 4^{\circ} \mathrm{C}$ Hold. We then added a Second Strand Master Mix to generate the second strand cDNA $\left(16^{\circ} \mathrm{C}\right.$ for $\left.2 \mathrm{~h}\right)$. The cDNA fragments were purified using a QIAquick PCR Purification Kit (QIAGEN), and these cDNA fragments were combined with an End Repair $\operatorname{Mix}\left(20^{\circ} \mathrm{C}\right.$ for $\left.30 \mathrm{~min}\right)$. Subsequently, the cDNA fragments were purified, the A-Tailing Mix was added, and the mixture was incubated for the ligation reaction at $20^{\circ} \mathrm{C}$ for $20 \mathrm{~min}$. The products were then run on a $2 \%$ agarose gel to select fragments within 300-350 bp. The gel was purified with a QIAquick Gel Extraction kit (QIAGEN). Several rounds of PCR amplification with a PCR Primer Cocktail and a PCR Master Mix were performed to enrich the cDNA fragments. The PCR products were then purified using Ampure XP Beads (AGENCOURT). The cDNA library was validated by two different methods to determine the average molecular length using the Agilent 2100 bioanalyzer instrument (Agilent DNA 1000 Reagents), and by real-time quantitative PCR (QPCR; TaqMan Probe). The qualified libraries were amplified on cBot to generate a cluster on the flowcell (TruSeq PE Cluster Kit V3-cBot-HS, Illumina), and the amplified flowcell were paired- end sequenced on the HiSeq 2000 System (TruSeq SBS KIT-HS V3, Illumina).

\section{Transcriptome Analysis}

Raw reads from twelve libraries were generated and were transformed from image data output into sequence data. Sequencing quality value (SQ) of the sequence data was in the range of 2-35. Raw reads with only adapters and unknown or low quality bases, were removed, and the following analyses were based on clean reads only. De novo assembly of RNA-seq was conducted using Trinity (http://trinityrnaseq.sourceforge. net/). The unigenes were generated by Trinity modules and the processes of sequence splicing and redundancy removing were employed. The unigenes were divided into two classes: clusters and singletons. Clusters (CL) are group of unigenes with a sequence similarity of greater than $70 \%$ among them. Distinct clustering was performed to cluster the assembled unigenes transcript sequences to identify the same gene or homolog using a hierarchical clustering approach involving TGICL-CAP3 and CD-HIT20. The alignment results were used to decide the sequence direction of the unigenes using TGICL 2.1 (http://sourceforge.net/projects/tgicl/files/tgicl\%20v2.1/) and Phrap Release 23.0 (http://www.phrap.org/). If the results of the databases conflicted with each other, a priority order of NR, Swiss-Prot, KEGG, and COG was followed when deciding the sequence direction of unigenes. When a unigene was unaligned in any of the above databases the ESTS software was used to decide the sequence direction from the $5^{\prime}$ end to the $3^{\prime}$ end.

\section{Differential Expression and Pathway Analysis}

The fragments per $\mathrm{kb}$ per million fragments (FPKM) of each unigene in each library were calculated using Cufflinks (http://cufflinks.cbcb.umd.edu/). Referring to Audic and Claverie (1997), we used a rigorous algorithm to identify differentially expressed genes (DEGs) between two samples, and the False Discovery Rate (FDR) as a statistical method in multiple hypotheses testing to correct for $p$-value to guarantee the low FDR. A very stringent cutoff, FDR $\leq 0.001$ and a fold change value of 2 , were used to identify DEGs. Tool edgeR23 was used to identify significantly up- and down-regulated genes on the read count values of genes.

A GO functional analysis and KEGG Pathway analysis were conducted on DEGs. First, all of the DEGs were BLASTed in the GO database (http://www.geneontology.org/) and the gene numbers were calculated for each GO term with GO-Term Finder v. 0.86 (http://search.cpan.org/dist/GO-TermFinder/). Then, a hyper geometric test was used to find significantly enriched GO terms in DEGs to compare with the genome background using the $p$-value. The calculated $p$-value was calibrated using the Bonferroni correction. GO terms were defined as significantly enriched GO terms in DEGs, if corrected $p \leq 0.05$. Pathway enrichment analysis identifies significantly enriched metabolic pathways or signal transduction pathways in DEGs when compared with the whole genome background. The calculated $p$-value for the pathway enrichment analysis was similar to that in the GO analysis. After multiple testing corrections, the pathways with a $p \leq 0.05$ were considered significantly enriched in DEGs.

\section{Quantitative Real-Time-PCR Analysis}

To validate the RNA-seq data, 14 genes were randomly selected to be analyzed by qRT-PCR with a reference gene (Actin; Table $\mathbf{1}$ ). Sample treatment and RNA isolation were obtained following previously described above. Three independent biological replicates of each sample and three technical replicates of each biological replicate were used in the RT-PCR using the ABI7500 Fast Real-Time PCR System (Applied Bio-systems, USA) with a 96-well block. The reverse-transcription reactions were performed using the iScript ${ }^{\mathrm{TM}}$ advanced cDNA Synthesis Kit (BIO-RAD). PCR amplifications were performed in $20 \mathrm{uL}$ total volume reactions containing $2.5 \mathrm{uL}$ templates, $10 \mathrm{uL}$

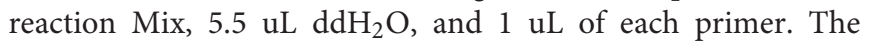
reaction conditions were $30 \mathrm{~s}$ at $95^{\circ} \mathrm{C}$, followed by 40 cycles of $95^{\circ} \mathrm{C}$ for $5 \mathrm{~s}$ and $60^{\circ} \mathrm{C}$ for $34 \mathrm{~s}$. The melting-curve was obtained by applying increasing temperature from 58 to $95^{\circ} \mathrm{C}$. To determine the relative fold change for each sample in each experiment the CT-value for the reference gene and six randomly chosen genes were calculated using the CT method as previously described (Livak and Schmittgen, 2001). 
TABLE 1 | Primer sequences used for Quantitative real-time-PCR analysis.

\begin{tabular}{|c|c|c|c|}
\hline Seq-ID & Gene name & Primer Sequence $\left(5^{\prime}-3^{\prime}\right)$ and $\operatorname{Tm}\left({ }^{\circ} \mathrm{C}\right)$ & GO-Cellular Component \\
\hline \multirow[t]{2}{*}{ AJ585201 } & Actin & F TCCTCACGCCATTCTT (59.10) & GO:0008372; cellular component \\
\hline & & R TCTCCTTGATGTCCCT (59.08) & \\
\hline \multirow[t]{2}{*}{ Unigene42814 } & Sterol 24-C-methyltransferase & F CGAACTTCAAGCACACTCGT (59.09) & GO:0009506//plasmodesma;GO:0005773// \\
\hline & & R CAGTACCCTITGGTGCAATG (59.05) & vacuole;GO:0005783//endoplasmic reticulum \\
\hline \multirow[t]{2}{*}{ Unigene42248 } & GDP-D-mannose 3', 5'-epimerase & F AATCCAACCATTCGGTCATT (59.10) & - \\
\hline & & R TACAAGCCGATGAGGCATAG (58.90) & \\
\hline \multirow[t]{2}{*}{ Unigene19563 } & Acyl-CoA dehydrogenase & F GCAACCAACATTGAAACGAG (59.17) & - \\
\hline & & R TGATGACGAGGCTTAGTTGG (58.87) & \\
\hline \multirow[t]{2}{*}{ CL1489 } & Predicted protein & F GCTATTACCGCAAGGTCCAT (59.07) & - \\
\hline & & R TCGATCATATGGCACAACAA (58.49) & \\
\hline \multirow[t]{2}{*}{ CL11729 } & Abscisic acid receptor & F GCTGCACTTCACCAAAGAAA (59.05) & - \\
\hline & PYR/PYL family & R TGCTCTCGAATTTCTCAACG (59.15) & \\
\hline \multirow[t]{2}{*}{ CL10698 } & Hexokinase & F TGGACCTAGGAGGGACAAAC (58.99) & GO:0005739//mitochondrion;GO:0009536//plastid;GO: \\
\hline & & R AACCAAACAATTCCGAGGAG (59.03) & $\begin{array}{l}\text { 0005773//vacuole;GO:0005634//nucleus;GO:0005886// } \\
\text { plasma membrane }\end{array}$ \\
\hline \multirow[t]{2}{*}{ Unigene30003 } & Hypothetical protein & F TCCGCAAACAAATCGTAGAG (58.92) & - \\
\hline & CHLNCDRAFT_56419 & R CAAAGACAGGACCAGCAGAA (59.01) & \\
\hline \multirow[t]{2}{*}{ CL12576 } & 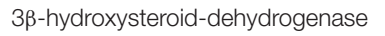 & F AAGCATACACGCAAACGAAG (58.99) & GO:0016020//membrane \\
\hline & & R CAAGTGCGCAGCTCATTTAT (59.09) & \\
\hline \multirow[t]{2}{*}{ Unigene48238 } & Uncharacterized protein & F GAAGAGGTATGCTGATGGCA (58.83) & GO:0005739//mitochondrion;GO:0009579// \\
\hline & LOC100501669 & R AGACCATACGCCTTGGTAGC (59.22) & $\begin{array}{l}\text { thylakoid;GO:0009507//chloroplast;GO:0016020// } \\
\text { membrane;GO:0005783//endoplasmic reticulum }\end{array}$ \\
\hline \multirow[t]{2}{*}{ Unigene2564 } & Hypothetical protein & F GCCGATCCAACTCATACCTT (59.01) & GO:0009941//chloroplast velope;O:0048046// \\
\hline & PHYSODRAFT_484706 & R TGCATGCTTGAGGATACCAT (59.10) & $\begin{array}{l}\text { apoplast;GO:0010319//stromule;GO:0005829//cytosol; } \\
\text { GO:0009570//chloroplaststroma;GO:0009579//thylakoid }\end{array}$ \\
\hline \multirow[t]{2}{*}{ Unigene3201 } & Shaggy-related protein kinase & F CTCGTAACACCGACACCATC (59.01) & GO:0005829//cytosol \\
\hline & & R CGTTGTCTAACATCCATCGG (59.00) & \\
\hline \multirow[t]{2}{*}{ Unigene28957 } & Predicted protein & F ACGACCAAAGTGGTGAACAA (59.03) & GO:0009941//chloroplast envelope;GO:0005794//Golgi \\
\hline & & R TGCCGAAATCAAGACACAAT (59.13) & apparatus;GO:0005783//endoplasmic reticulum \\
\hline \multirow[t]{2}{*}{ Unigene41363 } & Predicted protein & F CGACTACAAGGACTGGCAGA (59.03) & - \\
\hline & & R CAATACCACCCACAGCTTCA (59.57) & \\
\hline \multirow[t]{2}{*}{ Unigene21403 } & Aspartate aminotransferase & F GGGATGCATITGGAGATGA (59.39) & GO:0009507//chloroplast \\
\hline & & R TGAGCAGAGTCGTTGCTTCT (58.91) & \\
\hline \multirow[t]{2}{*}{ CL7857 } & Os08g0127100 & F TTCAGCCTCTCATGGTTCAC (58.80) & GO:0016021//integral to membrane; \\
\hline & & R GCCACGATCATCAGAATCAC (59.04) & GO:0005886//plasma membrane \\
\hline
\end{tabular}

\section{RESULTS}

\section{Drought-Induced Antioxidant Enzymes in Two L. multiflorum Lines and Their Responses to Drought Stress}

To investigate the effects of drought stress resulting in oxidative stress in two L. multiflorum lines, enzyme change was quantified after plants were treated with drought stress for 1 or $2 \mathrm{~h}$. The results showed that there was a significant difference in nontreated and drought-treated seedlings. Leaf transpiration rate and leaf water potential in two L. multiflorum lines after 1 and $2 \mathrm{~h}$ of treatment had significant difference compared with control, especially for susceptible lines (Figures 1A,B). As a major indicator of the stress-triggered ROS level oxidative damage (Badawi et al., 2004b; He et al., 2012), malondialdehvde (MDA) content was measured in well-watered and drought-treated plants. There were significant differences among the treated and control seedlings (Figure 1C). A lower $\mathrm{H}_{2} \mathrm{O}_{2}$ concentration in drought-tolerant lines could be due to the higher activity of the enzymatic activities of catalases (CAT), superoxide dismutase (SOD), dehydroascorbate reductase (DHAR), and monodehydroasorbate reductase (MDHAR; Figures 1D-H). These results indicated that ROS accumulation was increased by the development of an antioxidant defense system in two L. multiflorum lines exposed to a short-term drought. The resistant lines faced less oxidative damage than the susceptible lines.

\section{Read Generation and De novo Assembly}

The 12 RNA samples from two L. multiflorum lines were sequenced using the Illumina HiSeq 2000 platform, which generated 0.4 billion clean reads. After filtering for quality, a total of 39.8 million clean reads were assembled into 128,622 unigenes having an average length of 870 nt. Over $90 \%$ of the cleaned 


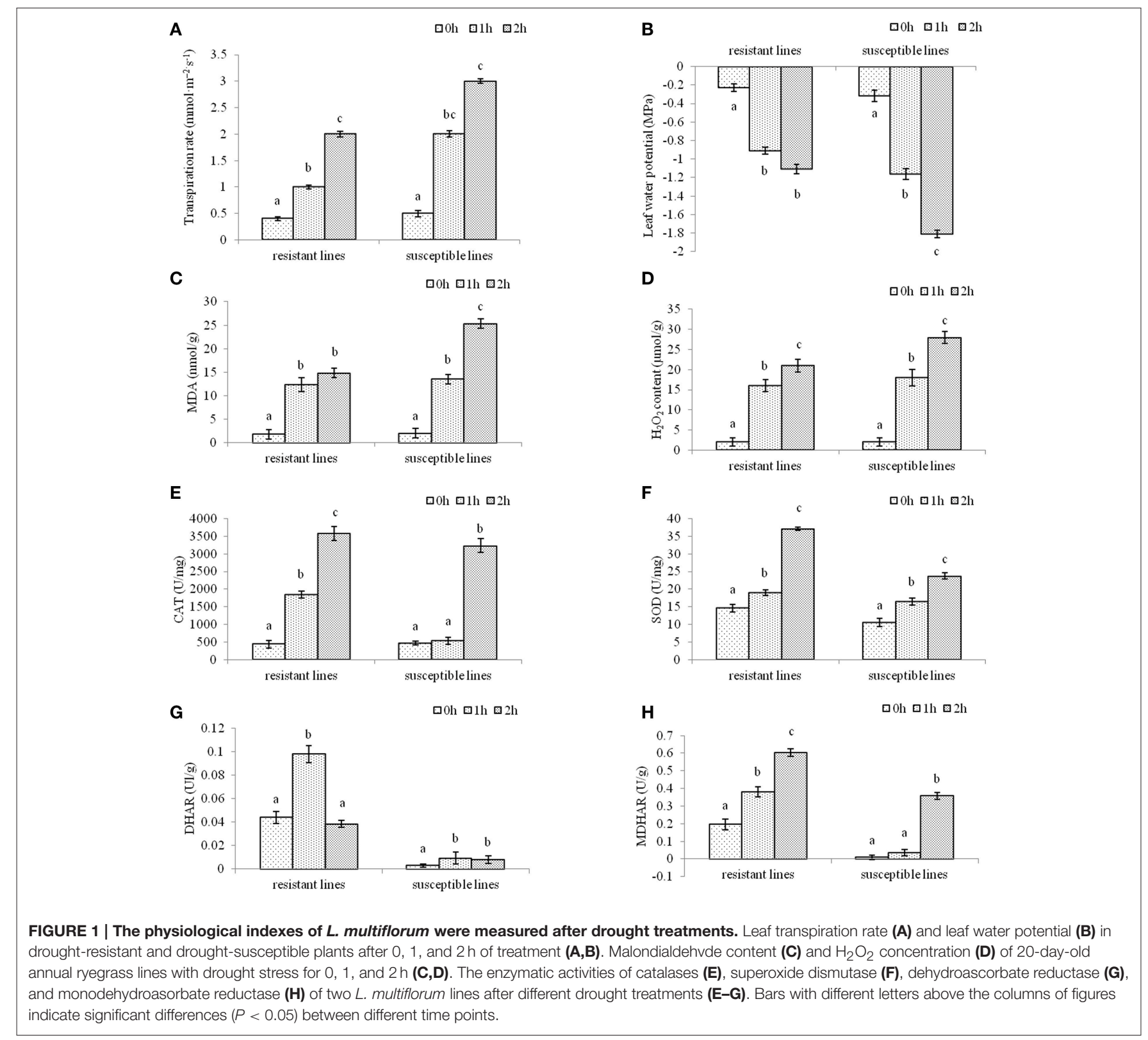

reads were able to be mapped to the assembly (Table 2; Accession nos: GSE78738 in the NCBI GEO database). In total, 600, 565 consensus sequences were generated after clustering the similar sequences with greater than $70 \%$ sequence similarity.

\section{Differential Expression and GO Enrichment Analysis}

To analyze drought-mediated gene expression of two L. multiflorum lines, a differential expression analysis was performed on resistant and susceptible lines. A total of 78,754 genes were significantly changed by treating plants with drought for 1 and $2 \mathrm{~h}$, of which 52,721 were upregulated genes and 26,033 were down-regulated genes (Figure 2A), Specific information regarding up-regulated and down-regulated genes is listed in Supplementary Table 1.
Moreover, a total of 4718 DEGs were identified by using NOlSeq analysis $(|\log \mathrm{FC}|>1$, Probability $>0.7$; Tarazona et al., 2011; Supplementary Figure 1). More genes were significantly up-regulated in the resistant lines when compared with the susceptible lines under short-term drought stress in Figures 2B,C).

A Gene Ontology (GO) analysis was conducted to reveal the biological processes of DEGs that were exposed to drought stress. A large number of DEGs related to metabolic processes such as carbohydrate metabolism, lip metabolism and signal transduction were identified (Figure 3A). The percentage of DGEs was used to evaluate the importance of these metabolic processes, suggesting that lipid metabolism, carbohydrate metabolism, and signal transduction are dramatically affected by drought (Figures 3C,D). The pathways that are known 
TABLE 2 | The numbers of mapped reads and assembled unigenes.

\begin{tabular}{|c|c|c|c|c|c|}
\hline Samples & Sequenced reads & Mapping ratio (\%) & Total consensus sequences & Distinct clusters & Distinct singetons \\
\hline Resistant-0 & $73,659,506$ & 92.0 & 105,900 & 35,928 & 69,972 \\
\hline Reisitant-1 & $71,287,722$ & 91.8 & 120,531 & 34,640 & 85,891 \\
\hline Resistant-2 & $73,094,738$ & 91.3 & 98,840 & 32,319 & 66,521 \\
\hline Susceptible-0 & $70,721,532$ & 92.0 & 96,611 & 31,201 & 65,410 \\
\hline Susceptible-1 & $72,176,332$ & 91.9 & 85,628 & 24,417 & 58,211 \\
\hline Susceptible-2 & $72,072,732$ & 92.3 & 93,055 & 28,477 & 64,608 \\
\hline Total & $433,012,562$ & & 600,565 & 53,829 & 410,613 \\
\hline
\end{tabular}

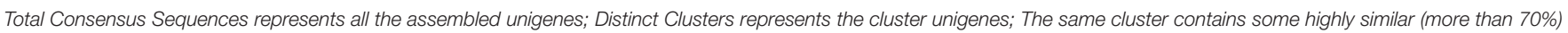

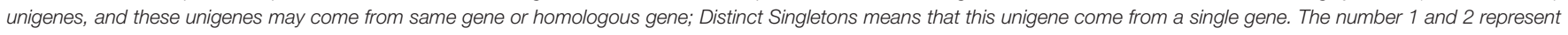
drought treatment time for 1 and $2 h$, respectively.
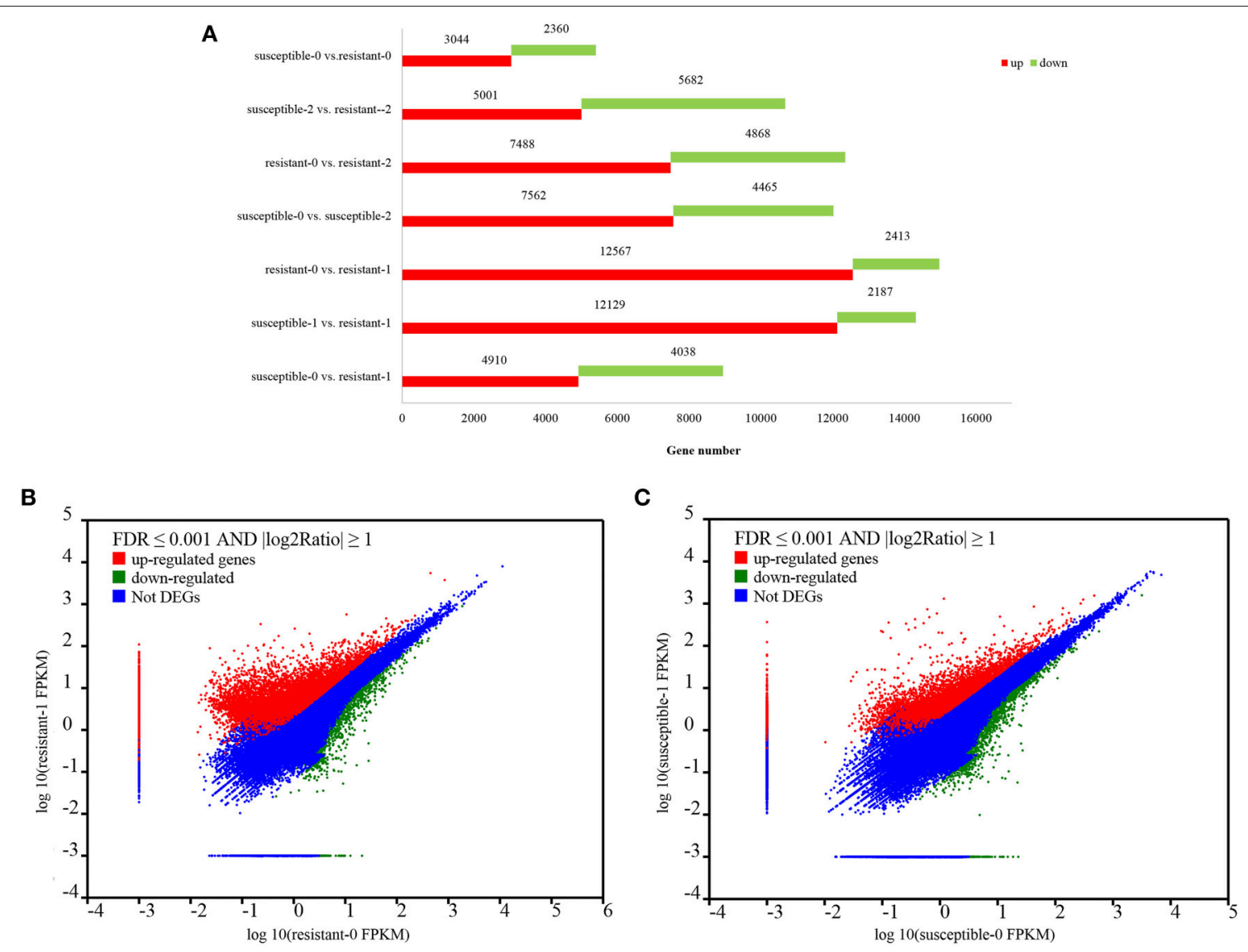

FIGURE 2 | Statistics of up- and down-regulated genes between the two L. multiflorum lines (A); The expression level of DEGs in resistant and susceptible lines exposed to drought (B-C). The upper with red regions reveal those genes with significantly up-regulated expression. Similarly, the lower with green dots shows the down-regulated genes and the blue dots represent the region with no DGEs.

to be involved in regulating mechanisms were differentially expressed under drought. Furthermore, the different functional characteristics of DEGs such as catalytic activity, transferase activity, binding, and structural constituent of the ribosome were discovered (Figure 3B).

Using GO enrichment analysis, the changes of transcript abundance of enriched genes were observed by comparing plants treated with drought 1 and $2 \mathrm{~h}$. For resistant lines, the number of enriched genes significantly decreased in terms of "response to osmotic stress" and "response to a water stimulus," whereas the opposite result was observed in susceptible lines (Figure 4A). The DEGs of "response to an organic substance" and "response to an oxygen-containing compound" were not significantly enriched in tolerant lines, but were dramatically enriched in susceptible lines (Figure 4B). The numbers of DEGs found in "response to lipids" and "nucleobase-containing compound biosynthetic 


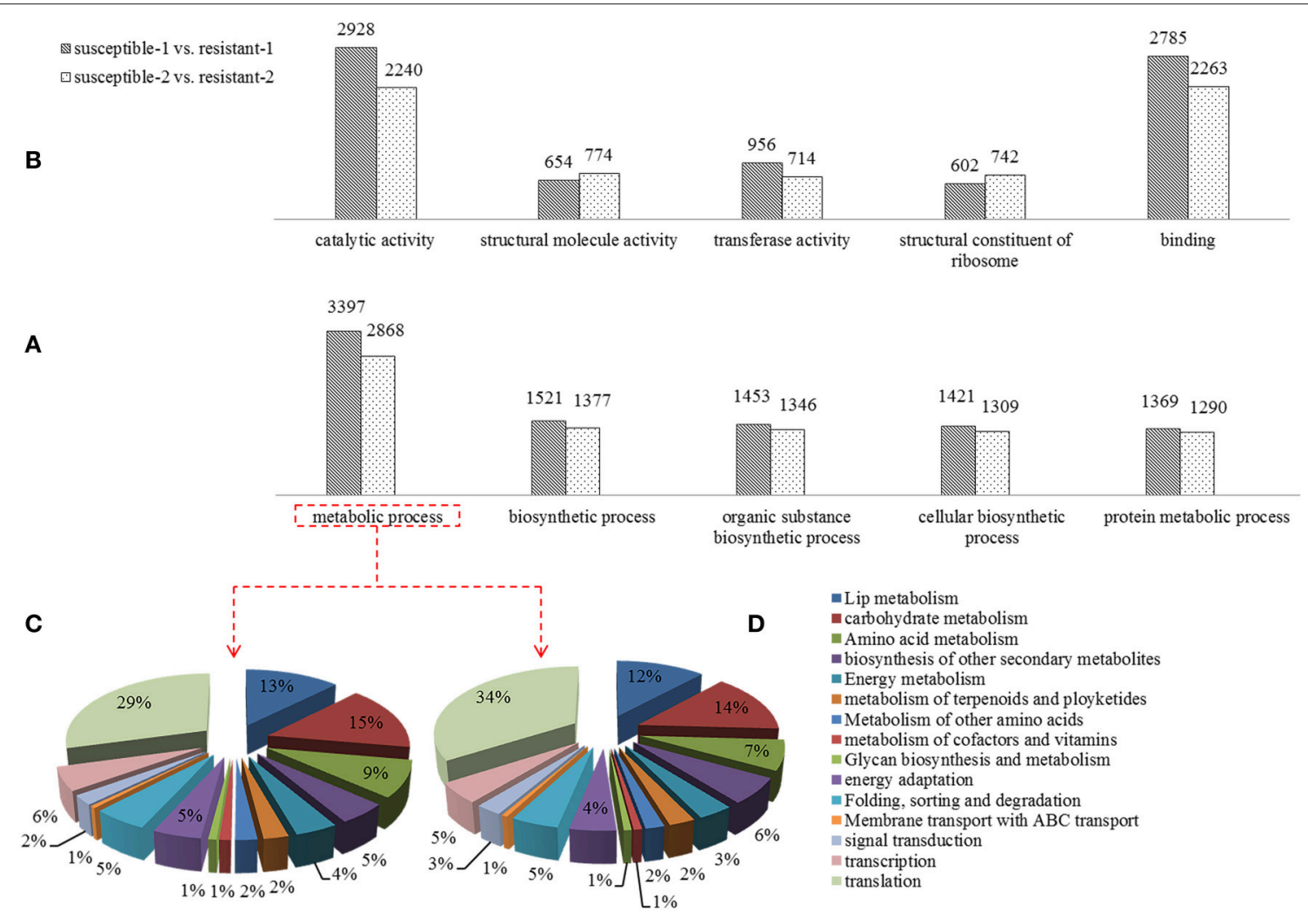

FIGURE 3 | Comparison of differentially expressed genes of the Gene Ontology (GO) analysis including biological process (A) and molecular functions (B) in two annual ryegrass lines subjected to drought stress for $\mathbf{1}$ and $\mathbf{2} \mathbf{h}$. And the percent of DEGs involved in metabolic processes in resistant and susceptible lines is shown in (C,D).

process" increased in the two L. multiflorum lines when treated with 1-2 h of drought stress (Figure 4C).

\section{Quantitative Real-Time-PCR Validation of Differentially Expressed Transcripts from RNA-Seq}

To confirm the RNA-Seq results, select genes that showed upand down-regulation in the two L. multiflorum lines were chosen for qRT-PCR. The relative gene expression of qRT-PCR was calculated using the $2^{-\Delta \Delta \mathrm{Ct}}$ method. The expression trends of these genes agreed with the RNA-Seq data (Figure 5), and a significant correlation of fold change was identified in resistant lines $\left(r^{2}=0.982\right)$ and susceptible lines $\left(r^{2}=0.814\right)$, suggesting that reliable RNA-Seq results were obtained in this study.

\section{Responses of Carbon Metabolism-Associated Genes to Drought Stress}

Glycolysis and gluconeogenesis pathways are primary metabolism pathways that may be modulated to establish a new homeostasis under drought stress in plants (Caruso et al., 2009). Changes in the expression levels of a large number of glycolysis and gluconeogenesis-associated genes in two L. multiflorum lines were observed (Figure 6A). Genes encoding enzymes, specifically glucose-6-phosphate isomerase (GPI) and probable phosphoglycerate mutase (PGAM) showed significant up-regulation in the resistant lines, whereas no change was observed in the susceptible lines. L-lactate dehydrogenase $(\mathrm{LDH})$ participated in up-regulation in resistant lines but was down-regulated in susceptible lines. A contrasting result was observed with phosphoglycerate kinase (PGK), which was down-regulated in resistant lines and up-regulated in susceptible lines. In addition, a large proportion of the genes encoding enzymes, fructose-bisphosphate aldolase (FBA), glyceraldehyde 3-phosphate dehydrogenase (GAPCs), pyruvate decarboxylase (PDC), dihydrolipoamide acetyltransferase (DLA), phosphoenolpyruvate carboxykinase (PEPC) showed up-regulated expression in the resistant lines. Drought induced a relatively large increase in the levels of glucose in resistant lines and resulted in a sharp increase in fructose, especially when plants were treated with drought stress for $1 \mathrm{~h}$, as shown in Supplementary Figures 2A,B. These results indicated that the activities of glycolysis and gluconeogenesis-associated genes may play a role in the response of resistant lines to drought.

Changes in the expression of several starch and sucrose metabolic genes in resistant lines were observed (Figure 6B). There were two genes annotated as encoding trehalose 6-phosphate synthase/phosphatase (TPP), and xylan 1, $4-\beta$-xylosidase, which are both involved in starch and sucrose 
A

response to water stimulus

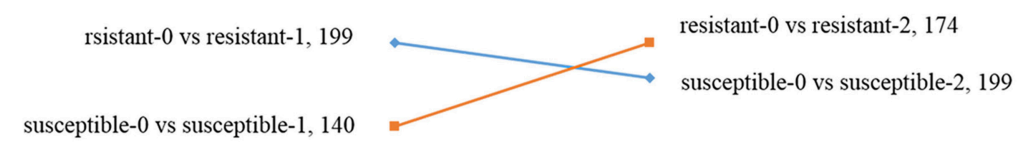

\section{response to osmotic stress}

rsistant- 0 vs resistant-1, 337

susceptible- 0 vs susceptible-1, 200

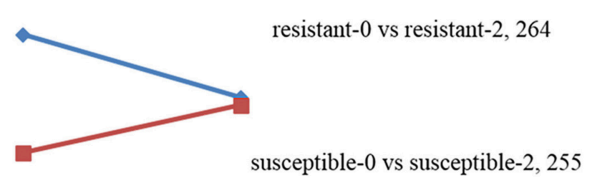

B

response to organic substance

rsistant-0 vs resistant-1, 525

susceptible-0 vs susceptible-1, 353

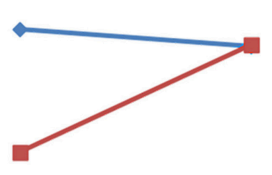

resistant-0 vs resistant-2, 501

susceptible-0 vs susceptible-2, 504

response to oxygen-containing compound

resistant -0 vs resistant- 2,559

rsistant-0 vs resistant-1, 564 susceptible- 0 vs susceptible-1, 427

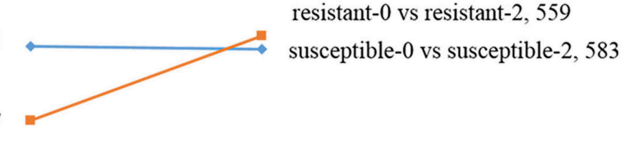

C

\section{response to lipid}

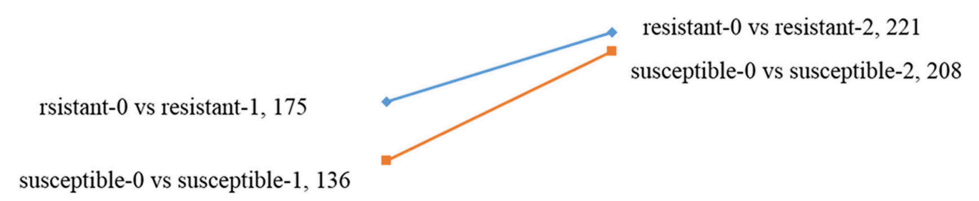

nucleobase-containing compound biosynthetic process

rsistant-0 vs resistant-1, 190

susceptible-0 vs susceptible-1, 169

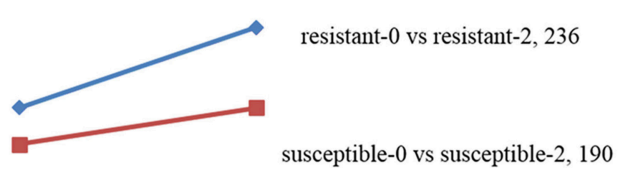

FIGURE 4 | Scatter diagrams of different changing trends of DEGs in two L. multiflorum lines by GO enrichment analysis after drought treatment for 1 and $\mathbf{2} \mathbf{h}$. The GO terms included response to osmotic stress and response to a water stimulus (A), response to an organic substance and response to an oxygen-containing compound (B), and response to lipid and nucleobase-containing compound biosynthetic process (C). 


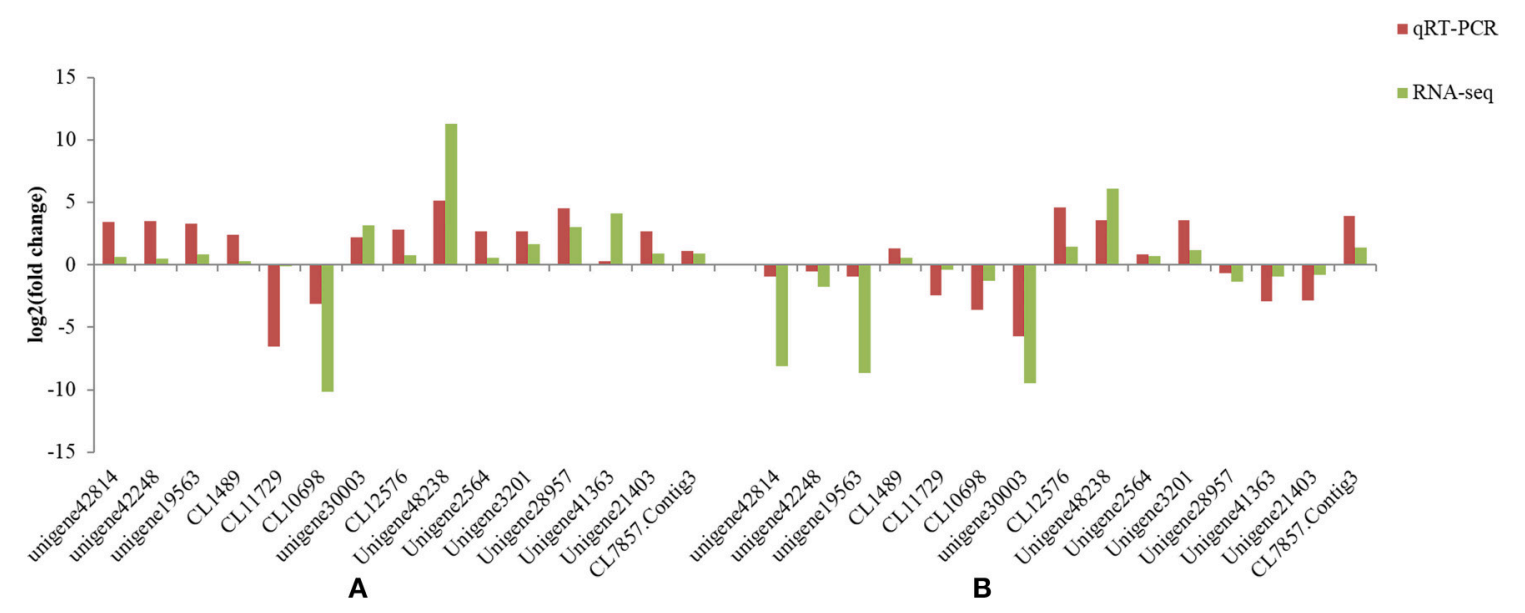

FIGURE 5 | Comparison of the different expression trends among 15 genes using both qRT-PCR results and RNA-Seq data in the resistant lines (A) and susceptible lines (B) of L. multiflorum.

metabolism, and which showed up-regulation in resistant lines and down-regulation in susceptible lines. The 1, 3$\beta$-glucan synthase, phosphoglucomutase (PGM), GPI, and starch phosphorylase (SP) participated in up-regulation in resistant lines, whereas no changes were observed in the susceptible lines.

\section{Lipid Metabolism Related to Gene Expression Response to Drought Stress}

A decrease in membrane lipid content was caused by the degradative processes that are induced by drought (De Paula et al., 1990). Lipids are important membrane components and the change in their composition may help to maintain membrane integrity and preserve cell compartmentation under water stress conditions. In response to drought stress, the lipid content in leaves decreased and fatty acids represented 61\% of contents in Arabidopsis thaliana (Gigon et al., 2004). Several DEGs related to glycerophospholipid metabolism have significant expression changes in the two L. multiflorum lines (Figure 6C). Genes related to gamma-glutamyltranspeptidase (GGT), glutathione reductase (GSR), L-ascorbate peroxidase (AP), and 6-phosphogluconate dehydrogenase (6-PGD) showed up-regulated expression in resistant lines, whereas these enzymes exhibited down-regulated expression in susceptible lines. The gene encoding enzyme glucose-6-phosphate 1-dehydrogenase $(\mathrm{G} 6 \mathrm{PDH})$ was obviously up-regulated in resistant lines, while the enzyme was not significantly up-or down-regulated in glycerophospholipid metabolism in the susceptible lines. For ether lipid metabolism, 1-alkyl-2-acetylglycerophosphocholine esterase was affected in the resistant lines but no changes were observed in the susceptible lines.

\section{Signal Transduction-Related Genes in Two L. multiflorum Lines}

Gene encoding hormone signal transduction enzymes may participate in several critical processes of plant morphogenesis such as cell division and enlargement, stem growth and stomatal closure (del Pozo and Ramirez-Parra, 2015). Under drought stress, a number of DEGs encoding transcriptional factors were identified in resistant lines (Figure 6D). Among them, 1-phosphatidylinositol-4-phosphate 5-kinase (EC 2.7.1.68), phosphatidylinositol phospholipase C (EC 3.1.4.11), 1D-myoInositol-tetrakisphosphate 5-kinase (EC 2.7.1.140), diacylglycerol kinase (DGK; EC 2.7.1.107), and 1-phosphatidylinositol-4phosphate 5-kinase (EC 2.7.1.68) exhibited up-regulation in the resistant lines. The remaining DEG encoded phosphatidate cytidylyltransferase (EC 2.7.7.41), which was observed as downregulated under drought stress. Furthermore, the LAX family gene, auxin influx carrier (AUX1), was up-regulated in resistant lines and down-regulated in the susceptible lines. In contrast, three of the drought-responsive proteins (jasmonic acid-amino synthetase, histidine-containing phosphotransfer protein, and regulatory protein NPR1) were observed up-regulated only in the resistant lines.

\section{DISCUSSION}

\section{The Response of Drought-Induced Antioxidant Enzymes to Oxidative Stress}

Oxidative damage remains a potential problem as it interrupts normal plant metabolism. The level of physiological response depends on the species, the stage of development, and the metabolic state of the plant, as well as the duration and intensity of the stress (Pastori et al., 2000). ROS are produced in plants under various stress conditions and serve as important mediators in plant responses to stresses. Oxidation stress has been found to enhance drought tolerance of plant species ( $\mathrm{Zhu}$, 2001). However, little is known about the genes that encode antioxidant involved in drought tolerance and antioxidants defenses in annual ryegrass to short-term drought conditions. Superoxide dismutase (SOD), dehydroascorbate reductase (DHAR), and monodehydroasorbate reductase (MDHAR) 


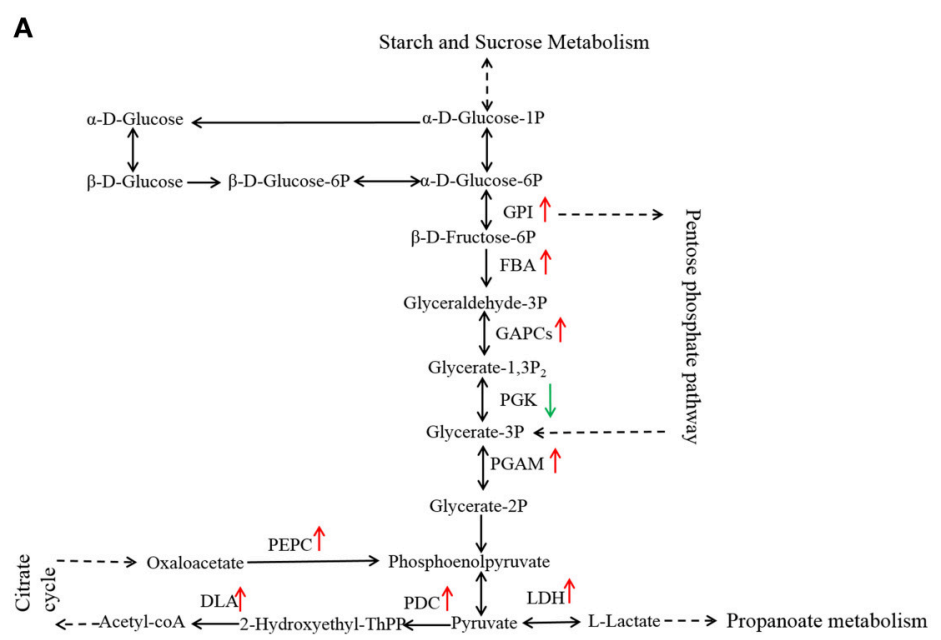

B

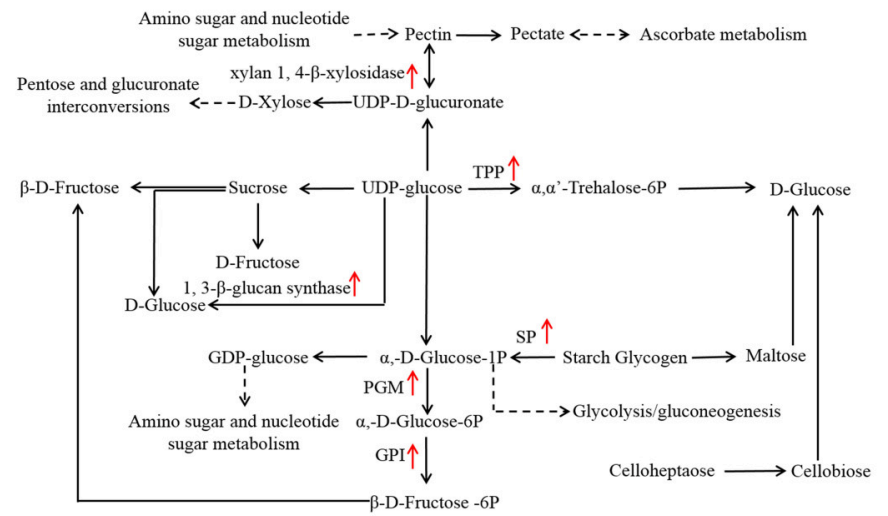

C

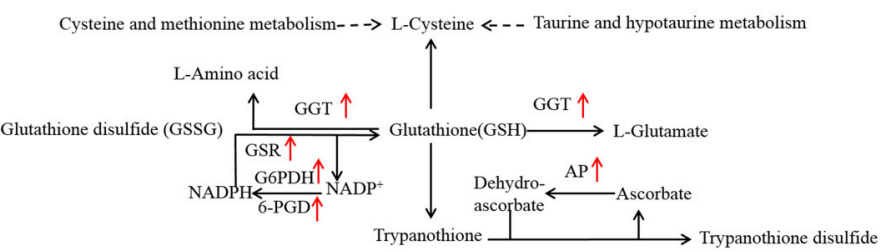

D

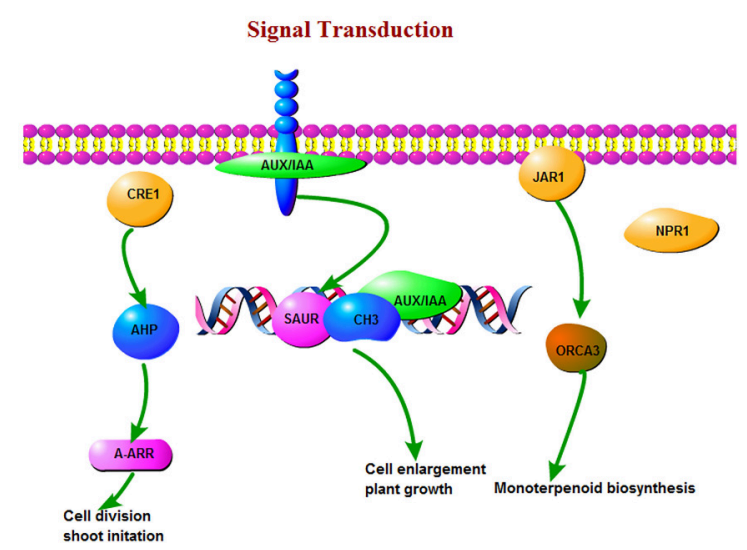

FIGURE 6 | Effects of drought stress on the expression of genes associated with glycolysis and gluconeogenesis (A), starch and sucrose metabolism (B), glycerophospholipid metabolism (C), and signal transduction (D) in resistant lines. The red arrow indicates significantly up-regulated expression. 
are the most effective intracellular enzymatic antioxidant, and they are important in plant stress tolerance by providing defense against the toxic effects of elevated levels of ROS (Gill and Tuteja, 2010). Catalases (CAT) are indispensable for the detoxification of ROS in stress inducing environments (Garg and Manchanda, 2009). In this study, resistant lines showed higher activity of the enzymatic activities of CAT, DHAR, and MDHAR than susceptible lines in both two time points subjected to high $\mathrm{H}_{2} \mathrm{O}_{2}$ content. But drought had no significant effect on activity of SOD and MAD content with drought for $1 \mathrm{~h}$. These results indicated that most of activities of antioxidant enzymes significantly increased to maintain cellular ROS for osmotic adaptation in drought resistant lines. Based on the result of the transcriptome, several genes encoding histidinecontaining phosphotransfer proteins (HPTs), GGT, L-ascorbate peroxidase (AP), 6-phosphogluconate dehydrogenase (6-PGD), and $\mathrm{G} 6 \mathrm{PDH}$ function as antioxidants and play an important role in increasing the tolerance of resistant lines against drought stress.

\section{Carbon Metabolism-Related Genes Contributed to Enhanced Drought Tolerance}

A change in glycolysis and gluconeogenesis is considered to be a basic characteristic of plant adaption to abiotic stress. Glycolysis is an important metabolic pathway in carbohydrate metabolism, and drought stress leads to altered sucrose and amino acid contents, which was revealed by metabolite analysis (Broeckling et al., 2005). Genes encoding enzymes showed significant regulation in glycolysis and gluconeogenesis metabolism and the results are consistent with a droughtmediated function in photosynthetic carbon metabolism (Kovács et al., 2006; Shaar-Moshe et al., 2015). In our study, several key enzymes in glycolysis/gluconeogenesis metabolism were differentially expressed in resistant and susceptible lines under drought stress. Genes encoding hexokinase (HxK) showed up-regulation in resistant lines. The activity of $\mathrm{HxK}$ is expected to be critical to the cellular levels of glucose and fructose, and the reactions catalyzed by HxK lead to hexoses entering the glycolytic pathway. In cereal crops, it has been shown that HxK decreased at the transcript levels in wheat and rice under drought stress (Xue et al., 2008; Lenka et al., 2011), but in maize HXK was found to be up-regulated under drought-stress after recovery irrigation (HayanoKanashiro et al., 2009). Moreover, putative phosphoglycerate mutase (PGAM) as a potential target is regulated by an identified miRNA in the glycolysis pathway (Guzman et al., 2012). The abundance of PGAM decreased under water deficit (Shu et al., 2011; Cramer et al., 2013). In our study, PGAM was significantly up-regulated in the resistant lines, suggesting that the protein might contribute to enhanced drought tolerance. L-lactate dehydrogenase (LDH) belongs to one of the most intensely studied enzyme families. Llactate dehydrogenase is a hydrogen transfer enzyme that catalyzes the reversible reaction of pyruvate to lactate, with nicotinamide-adenine dinucleotide $(\mathrm{NADH})$ as the hydrogen donor. Genes encoding LDH were up-regulated in the resistant lines of annual ryegrass. This is consistent with the results obtained through oxidative stress studies in Saccharomyces cerevisiae (Zhao et al., 2015). FBA is a constituent of both the glycolysis/gluconeogenesis pathway and the pentose phosphate cycle in plants (Konishi et al., 2004). FBA was up-regulated in the drought resistant lines, which validates the findings of Gong et al. (2010), who detected FBA in drought tolerant lines of tomato. It is interesting that we identified genes encoding glyceraldehyde-3-phosphate dehydrogenases (GAPCs) in glycolysis pathways that are up-regulated in drought tolerant lines, as reported in Arabidopsis. Glyceraldehyde-3-phosphate dehydrogenases may provide a direct connection between membrane lipid-based signaling, energy metabolism and growth control in a plant's response to ROS and water stress (Guo et al., 2012). Phosphoenol pyruvate carboxylase (PEPC) has been shown to participate in stress responses of $\mathrm{C} 4$ plants and C3 plants, as it served to balance carbon and nitrogen metabolism (Doubnerová and Ryšlavá, 2011). Despite the studies that have tried to understand the regulation and roles of PEPC, the role that this enzyme plays in drought stressed plants is unknown. PEPC might be involved in drought tolerance via alleviating the inhibitory effect of drought stress on photosynthesis (Bao-Yuan et al., 2011). In our study, upregulation of PEPC could possibly improve drought tolerance by affecting the metabolism of glycolysis and gluconeogenesis. Additionally, other genes involved in the glycolysis and gluconeogenesis pathways were not previously reported to be associated with drought response. Therefore, further studies are needed to understand the mechanism modulating plant growth under stress.

Similarly, we investigated the effect of drought on the activities of DEGs associated with starch and sucrose metabolism. Trehalose 6-P phosphatase (TPP) was a key enzyme that significantly enhanced drought resistance in a variety of organisms (Pilon-Smits et al., 1998). Interestingly, transgenic plants that express TPP genes from microorganisms not only exhibit an increase in drought tolerance, but also show strong developmental alterations (Jang et al., 2003). In the current study, the up-regulation of TPP may have played a role in the response of resistant lines to drought, thus resulting in changes in carbohydrate allocation and metabolism (Redillas et al., 2012). Phosphoglucomutase (PGM) contributes to the coordination of sucrose synthesis with the rate of carbon dioxide fixation, and to the control of partitioning of photosynthate between sucrose and starch (Toldi et al., 2002; Nielsen et al., 2004). There was a slight decrease in PGM in drought-stressed wheat leaves (Zhu, 2001). However, we observed an up-regulation of PGM in the drought resistant lines of annual ryegrass, which agreed with the findings of Pinheiro et al. (2008). GPI has been shown to fill unique roles in carbohydrate metabolism during drought stress in different plants, e.g., Arabidopsis (Seki et al., 2002) and wheat (Xue et al., 2013). In seedling development under water stress, the decrease in the expression of starch phosphorylase might be due to inhibitory effects on starch concentrations (Peng et al., 2014). Compared with the drought sensitive lines, the drought tolerant line maintained higher 
activity of leaf starch phosphorylase in the chickpea (Awasthi et al., 2014). Inconsistent with our results, drought affected the activity of starch phosphorylase in annual ryegrass and showed up-regulated expression in the drought resistant lines. The change in the up-regulated expression of genes related to starch and sucrose metabolism after drought stress was striking, indicating that drought resistant annual ryegrass lines invests more energy and resources into immediate defense needs than the susceptible lines.

\section{Lipid Metabolism-Related Genes and Drought-Induced Genes Used as Antioxidants}

Under drought conditions, considerable changes in lipid metabolism were observed (Benhassaine-Kesri et al., 2002). Previous studies have investigated responsive genes of lipid metabolism in ryegrass (Foito et al., 2013), but the results have not been previously linked with annual ryegrass's response to drought stress. We identified five related up-regulated genes in drought resistant lines under drought stress. Genes encoding glutathione reductase (GSR) and GGT were identified as important enzymes in glycerophospholipid metabolism. The down-regulation of the GGT gene might play a negative regulatory role in GSH degradation and the GSR gene may have been up-regulated when subjected to drought stress, which consequently mitigated the oxidative stress (Fan et al., 2014). In the present study, we observed up-regulation of genes encoding GSR and GGT, suggesting that the two enzymes at least partially contribute to the high drought tolerance in annual ryegrass. It has been widely reported that L-ascorbate peroxidase (AP) participated in the ascorbate-glutathione cycle, which is an important process for free radical detoxification (Cramer et al., 2013). Significant up-regulation of the gene encoding AP was identified in this study; therefore, AP activity may influence drought tolerance by regulating glycerophospholipid metabolism and the ascorbate pathway in drought resistant lines to adapt to water deficit. In plants, a6-phosphogluconate dehydrogenase (6-PGD) and G6PDH are major sources of NADPH in the cytoplasm of plant cells. These key enzymes are also used for the detoxification of hydrogen peroxide through the ascorbate-glutathione cycle. A previous study found that the activities of 6-PGD and G6PDH increased when plants were subjected to many artificial or transitory stressors, except in reed (Phragmites communis) under longterm drought stress (Chen et al., 2003). This conclusion holds true for short-term drought stress adaptation in annual ryegrass.

\section{Signal Transduction-Related Genes and Transcription Factors Associated with Drought Tolerance}

Plants perceive environmental signals and transmit the signals to the cellular machinery to activate adaptive response in plants under abiotic stress (Xiong et al., 2002). Signal transduction requires certain molecules that participate in the modification, delivery, and assembly of signaling components (Ji et al.,
2013). Genes encoding 1-phosphatidylinositol-4-phosphate 5kinase (PIP kinase), which is involved in the defense response in poplars (Chen and Cao, 2015), plays a signaling role and the enzyme is a component of a signaling pathway associated with the synthesis of phosphatidylinositol phosphate (Gupta et al., 2013). Phosphatidylinositol-specific phospholipase C (PIase C) plays a central role in the phosphatidylinositol-specific signal transduction pathway. Georges et al. (2009) found that the overexpression of PIase $\mathrm{C}$ in canola enhances drought tolerance and promotes early flowering and maturation. Therefore, the up-regulation of PIase C may dramatically influence drought tolerance by regulating signal transduction in drought resistant annual ryegrass lines. 1D-myo-Inositol-tetrakisphosphate 5kinase has different expression in drought resistant and drought susceptible lines, but the specific molecular function of the enzyme is not clear. Phosphatidic acid (PA) plays a pivotal role in the plant's response to environmental signals (Arisz et al., 2009). PA can also be generated by DGK, which is thought to act as a switch with two functional implications: the termination of diacylglycerol (DAG) signaling and the initiation of PA signaling (Frere and Di Paolo, 2009). DGK has been reported in several plant species including tobacco, wheat, tomato, and Arabidopsis (Ge et al., 2012). In our study, up-regulated DGKencoding genes have been found in the drought resistant lines of annual ryegrass. In addition, phosphatidate cytidylyltransferase catalyzes the synthesis reaction of triglycerides, which are mainly found in cell membranes (Longmuir and Johnston, 1980). It is evident that the expression of genes coding phosphatidate cytidylyltransferase played an important role in the induction of drought resistance in citrus fruits (Ballester et al., 2011).

In our study, some of the DEGs encoding transcriptional factors were identified in the drought tolerant line. A previous study has demonstrated that AUX1in Arabidopsis encodes a high-affinity auxin influx carrier, and Arabidopsis AUX/LAX genes encode a family of auxin influx transporters that perform distinct developmental functions and have evolved distinct regulatory mechanisms (Péret et al., 2012). Furthermore, auxin influx results in enhanced resistance and the improvement of plant growth (Remy et al., 2013). Our results also give evidence to support this. Nonexpressorofpathogenesis_relatedgenes1 (NPR1) is known to be involved in salicylic acid (SA)mediated suppression of the jasmonic acid (JA) signaling pathway (Caarls et al., 2015). NPR1 is a regulator of basal and systemic acquired resistance in plants (Shimobayashi et al., 2013). Srinivasan et al. (2009) reported that Arabidopsis NPR1 enhances oxidative stress tolerance in transgenic tobacco plants. These results may explain why the expression of NPR1 genes was implicated in the drought tolerance of annual ryegrass. Histidine-containing phosphotransfer proteins (HPTs) have already been identified in the cytokinin transduction pathway (Hwang et al., 2002), which was isolated in a study on plant response to osmotic stress, salt stress, and drought stress (Chefdor et al., 2006). The HPTs were shown to up-regulate under drought stress and may help drought resistant ryegrass to withstand environmental stress. The genes involved in the signal transduction pathway 
contributed to the increase of drought tolerance in annual ryegrass.

\section{AUTHOR CONTRIBUTIONS}

LP collected data and wrote the manuscript. XZ and XM designed the project. MZ served as scientific advisors. JW, XM, and $\mathrm{LH}$ participated in technical editing of the manuscript. Other authors improved resolution of images.

\section{ACKNOWLEDGMENTS}

This research work was funded by the National Basic Research Program (973 program) in China (No 2014CB138705), the earmarked fund for Modern Agro-industry Technology Research
System (No.CARS-35-05) and the National Natural Science Foundation of China (NSFC 31372363).

\section{SUPPLEMENTARY MATERIAL}

The Supplementary Material for this article can be found online at: http://journal.frontiersin.org/article/10.3389/fpls.2016. 00519

\section{Supplementary Table 1 | List of partial RNA-seq-identified gens in L. multiflorum.}

Supplementary Figure 1 | Hierarchical clustering of differentially expressed genes.

Supplementary Figure 2 | Contents of glucose (A) and fructose (B) in two L. multiflorum lines subjected to drought stress for $\mathbf{1}$ and $\mathbf{2 h}$. Each value represents the mean $\pm \operatorname{SE}(n=4)$. The different letters indicate significant differences $(P<0.05)$ between different time points.

\section{REFERENCES}

Antolin, M., Yoller, J., and Sanchez-Diaz, M. (1995). Effects of temporary drought on nitrate-fed and nitrogen-fixing alfalfa plants. Plant Sci. 107, 159-165. doi: 10.1016/0168-9452(95)04108-7

Arisz, S. A., Testerink, C., and Munnik, T. (2009). Plant PA signaling via diacylglycerol kinase. Biochim. Biophys. Acta 1791, 869-875. doi: 10.1016/j.bbalip.2009.04.006

Aroca, R., Vernieri, P., Irigoyen, J. J., Sànchez-Dìz, M., Tognoni, F., and Pardossi, A. (2003). Involvement of abscisic acid in leaf and root of maize (Zea mays L.) in avoiding chilling-induced water stress. Plant Sci. 165, 671-679. doi: 10.1016/S0168-9452(03)00257-7

Audic, S., and Claverie, J. M. (1997). The significance of digital gene expression profiles. Genome Res. 7, 986-995

Awasthi, R., Kaushal, N., Vadez, V., Turner, N. C., Berger, J., Siddique, K. H., et al. (2014). Individual and combined effects of transient drought and heat stress on carbon assimilation and seed filling in chickpea. Funct. Plant Biol. 41, 1148-1167. doi: 10.1071/FP13340

Badawi, G. H., Kawano, N., Yamauchi, Y., Shimada, E., Sasaki, R., Kubo, A., et al. (2004a). Over-expression of ascorbate peroxidase in tobacco chloroplasts enhances the tolerance to salt stress and water deficit. Physiol. Plant. 121, 231-238. doi: 10.1111/j.0031-9317.2004.00308.x

Badawi, G. H., Yamauchi, Y., Shimada, E., Sasaki, R., Kawano, N., Tanaka, K., et al. (2004b). Enhanced tolerance to salt stress and water deficit by overexpressing superoxide dismutase in tobacco (Nicotiana tabacum) chloroplasts. Plant Sci. 166, 919-928. doi: 10.1016/j.plantsci.2003.12.007

Ballester, A. R., Lafuentes, M. T., Forment, J., Gadea, J., De Vos, R. C., Bovy, A., et al. (2011). Transcriptomic profiling of citrus fruit peel tissues reveals fundamental effects of phenylpropanoids and ethylene on induced resistance. Mol. Plant Pathol. 12, 879-897. doi: 10.1111/j.1364-3703.2011.00721.x

Bao-Yuan, Z., Zai-Song, D., and Ming, Z. (2011). Alleviation of drought stress inhibition on photosynthesis by overexpression of PEPC in rice. Acta Agron. Sin. 37, 112-118. doi: 10.1016/S1875-2780(11)60004-7

Benhassaine-Kesri, G., Aid, F., Demandre, C., Kader, J. C., and Mazliak, P. (2002). Drought stress affects chloroplast lipid metabolism in rape (Brassica napus) leaves. Physiol. Plant. 115, 221-227. doi: 10.1034/j.1399-3054.2002.1150207.x

Bewley, J. D., and Black, M. (1994). "Dormancy and the control of germination," in Seeds: Physiology of Development and Germination, 2nd Edn., eds J. D. Bewley and M. Black (New York, NY: Plenum Press), 199-271.

Białecka, B., and Kêpczyñski, J. (2010). Germination, $\alpha$-, $\beta$-amylase and total dehydrogenase activities of Amaranthus caudatus seeds under water stress in the presence of ethephon or gibberellin A3. Acta Biol. Cracov. Ser. Bot. 52, 7-12. doi: 10.2478/v10182-010-0001-0

Boyer, J. S. (1970). Leaf enlargement and metabolic rates in corn, soybean, and sunflower at various leaf water potentials. Plant Physiol. 46, 233-235. doi: $10.1104 /$ pp.46.2.233

Broeckling, C. D., Huhman, D. V., Farag, M. A., Smith, J. T., May, G. D., Mendes, P., et al. (2005). Metabolic profiling of medicago truncatula cell cultures reveals the effects of biotic and abiotic elicitors on metabolism. J. Exp. Bot. 56, 323-336. doi: $10.1093 /$ jxb/eri058

Caarls, L., Pieterse, C. M., and Van Wees, S. C. (2015). How salicylic acid takes transcriptional control over jasmonic acid signaling. Front. Plant Sci. 6:170. doi: 10.3389/fpls.2015.00170

Caruso, G., Cavaliere, C., Foglia, P., Gubbiotti, R., Samperi, R., and Laganà, A. (2009). Analysis of drought responsive proteins in wheat (Triticum durum) by 2D-PAGE and MALDI-TOF mass spectrometry. Plant Sci. 177, 570-576. doi: 10.1016/j.plantsci.2009.08.007

Chefdor, F., Bénédetti, H., Depierreux, C., Delmotte, F., Morabito, D., and Carpin, S. (2006). Osmotic stress sensing in Populus: components identification of a phosphorelay system. FEBS Lett. 580, 77-81. doi: 10.1016/j.febslet.2005.11.051

Chen, K. M., Gong, H. J., Chen, G. C., Wang, S. M., and Zhang, C. L. (2003). Up-regulation of glutathione metabolism and changes in redox status involved in adaptation of reed (Phragmites communis) ecotypes to drought-prone and saline habitats. J. Plant Physiol. 160, 293-301. doi: 10.1078/0176-1617-00927

Chen, M., and Cao, Z. (2015). Genome-wide expression profiling of microRNAs in poplar upon infection with the foliar rust fungus Melampsora larici-populina. BMC Genomics 16:696. doi: 10.1186/s12864-015-1891-8

Cramer, G. R., Van Sluyter, S. C., Hopper, D. W., Pascovici, D., Keighley, T., and Haynes, P. A. (2013). Proteomic analysis indicates massive changes in metabolism prior to the inhibition of growth and photosynthesis of grapevine (Vitis vinifera L.) in response to water deficit. BMC Plant Biol. 13:49. doi: 10.1186/1471-2229-13-49

Da Silva, J. V., Naylor, A. W., and Kramer, P. J. (1974). Some ultrastructural and enzymatic effects of water stress in cotton (Gossypium hirsutum L.) leaves. Proc. Natl. Acad. Sci. U.S.A. 71, 3243-3247. doi: 10.1073/pnas.71.8.3243

del Pozo, J. C., and Ramirez-Parra, E. (2015). Whole genome duplications in plants: an overview from Arabidopsis. J. Exp. Bot. 66, 21. doi: 10.1093/jxb/erv432

De Paula, F. M., Thi, A. P., De Silva, J. V., Justin, A., Demandre, C., and Mazliak, P. (1990). Effects of water stress on the molecular species composition of polar lipids from Vigna unguiculata L. leaves. Plant Sci. 66, 185-193. doi: 10.1016/0168-9452(90)90203-Z

Doubnerová, V., and Ryšlavá, H. (2011). What can enzymes of C 4 photosynthesis do for C 3 plants under stress? Plant Sci. 180, 575-583. doi: 10.1016/j.plantsci.2010.12.005

Eltayeb, A. E., Kawano, N., Badawi, G. H., Kaminaka, H., Sanekata, T., Shibahara, T., et al. (2007). Overexpression of monodehydroascorbate reductase in transgenic tobacco confers enhanced tolerance to ozone, salt and polyethylene glycol stresses. Planta 225, 1255-1264. doi: 10.1007/s00425-006-0417-7

Fan, Q.-J., Yan, F.-X., Qiao, G., Zhang, B. X., and Wen, X. P. (2014). Identification of differentially-expressed genes potentially implicated in drought response in pitaya (Hylocereus undatus) by suppression subtractive hybridization and cDNA microarray analysis. Gene 533, 322-331. doi: 10.1016/j.gene.2013.08.098 
Foito, A., Byrne, S. L., Hackett, C. A., Hancock, R. D., Stewart, D., and Barth, S. (2013). Short-term response in leaf metabolism of perennial ryegrass (Lolium perenne) to alterations in nitrogen supply. Metabolomics 9, 145-156. doi: 10.1007/s11306-012-0435-3

Frere, S. G., and Di Paolo, G. (2009). A lipid kinase controls the maintenance of dendritic spines. EMBO J. 28, 999-1000. doi: 10.1038/emboj.2009.77

Garg, N., and Manchanda, G. (2009). ROS generation in plants: boon or bane? Plant Biosyst. 143, 81-96. doi: 10.1080/11263500802633626

Ge, H., Chen, C., Jing, W., Zhang, Q., Wang, H., Wang, R., et al. (2012). The rice diacylglycerol kinase family: functional analysis using transient RNA interference. Front. Plant Sci. 3:60. doi: 10.3389/fpls.2012.00060

Georges, F., Das, S., Ray, H., Bock, C., Nokhrina, K., Kolla, V. A., et al. (2009). Over-expression of Brassica napus phosphatidylinositol-phospholipase C2 in canola induces significant changes in gene expression and phytohormone distribution patterns, enhances drought tolerance and promotes early flowering and maturation†. Plant Cell Environ. 32, 1664-1681. doi: 10.1111/j.13653040.2009.02027.x

Gigon, A., Matos, A.-R., Laffray, D., Zuily-Fodil, Y., and andPham-Thi, A. T. (2004). Effect of drought stress on lipid metabolism in the leaves of Arabidopsis thaliana (ecotype Columbia). Ann. Bot. 94, 345-351. doi: 10.1093/aob/mch150

Gill, S. S., and Tuteja, N. (2010). Reactive oxygen species and antioxidant machinery in abiotic stress tolerance in crop plants. Plant Physiol. Biochem. 48, 909-930. doi: 10.1016/j.plaphy.2010.08.016

Gong, P., Zhang, J., Li, H., Yang, C., Zhang, C., Zhang, X., et al. (2010). Transcriptional profiles of drought-responsive genes in modulating transcription signal transduction, and biochemical pathways in tomato. J. Exp. Bot. 61, 3563-3575. doi: 10.1093/jxb/erq167

Guo, L., Devaiah, S. P., Narasimhan, R., Pan, X., Zhang, Y., Zhang, W., et al. (2012). Cytosolic glyceraldehyde-3-phosphate dehydrogenases interact with phospholipase $\mathrm{D} \delta$ to transduce hydrogen peroxide signals in the Arabidopsis response to stress. Plant Cell 24, 2200-2212. doi: 10.1105/tpc.111.094946

Gupta, A., Toscano, S., Trivedi, D., Jones, D. R., Mathre, S., Clarke, J. H., et al. (2013). Phosphatidylinositol 5-phosphate 4-kinase (PIP4K) regulates TOR signaling and cell growth during Drosophila development. Proc. Natl. Acad. Sci. U.S.A. 110, 5963-5968. doi: 10.1073/pnas.1219333110

Guzman, F., Almerão, M. P., Körbes, A. P., Loss-Morais, G., and Margis, R. (2012). Identification of microRNAs from Eugenia uniflora by highthroughput sequencing and bioinformatics analysis. PLOS ONE 11:e49811. doi: 10.1371/journal.pone.0049811

Hayano-Kanashiro, C., Calderón-Vázquez, C., Ibarra-Laclette, E., Herrera-Estrella, L., and Simpson, J. (2009). Analysis of gene expression and physiological responses in three Mexican maize landraces under drought stress and recovery irrigation. PLoS ONE 4:e7531. doi: 10.1371/journal.pone.0007531

He, H., Xu, J., Xu, Y., Zhang, C., Wang, H., He, Y., et al. (2012). Cardioprotective effects of saponins from Panax japonicus on acute myocardial ischemia against oxidative stress-triggered damage and cardiac cell death in rats. J. Ethnopharmacol. 140, 73-82. doi: 10.1016/j.jep.2011.12.024

Hwang, I., Chen, H.-C., and Sheen, J. (2002). Two-component signal transduction pathways in Arabidopsis. Plant Physiol. 129, 500-515. doi: 10.1104/pp.005504

Jang, I.-C., Oh, S.-J., Seo, J.-S., Choi, W. B., Song, S. I., Kim, C. H., et al. (2003). Expression of a bifunctional fusion of the Escherichia coli genes for trehalose-6phosphate synthase and trehalose-6-phosphate phosphatase in transgenic rice plants increases trehalose accumulation and abiotic stress tolerance without stunting growth. Plant Physiol. 131, 516-524. doi: 10.1104/pp.007237

Ji, H., Greening, D. W., Barnes, T. W., Lim, J. W., Tauro, B. J., Rai, A., et al. (2013). Proteome profiling of exosomes derived from human primary and metastatic colorectal cancer cells reveal differential expression of key metastatic factors and signal transduction components. Proteomics 13, 1672-1686. doi: 10.1002/pmic.201200562

Konishi, H., Yamane, H., Maeshima, M., and Komatsu, S. (2004). Characterization of fructose-bisphosphate aldolase regulated by gibberellin in roots of rice seedling. Plant Mol. Biol. 56, 839-848. doi: 10.1007/s11103-004-5920-2

Kovács, G., Sorvari, S., Scott, P., and Toldi, O. (2006). Pyrophosphate: fructose 6-phosphate 1-phosphotransferase operates in net gluconeogenic direction in taproots of cold and drought stressed carrot plants. Acta Biol. Szeged. 50, 25-30. doi: 10.1556/AAgr.55.2007.1.8

Lan, P., Li, W., and Schmidt, W. (2012). Complementary proteome and transcriptome profiling in phosphate-deficient Arabidopsis roots reveals multiple levels of gene regulation. Mol. Cell. Proteomics 11, 1156-1166. doi: 10.1074/mcp.M112.020461

Lenka, S. K., Katiyar, A., Chinnusamy, V., and Bansal, K. C. (2011). Comparative analysis of drought-responsive transcriptome in Indica rice genotypes with contrasting drought tolerance. Plant Biotechnol. J. 9, 315-327. doi: 10.1111/j.1467-7652.2010.00560.x

Liu, Z., Li, Y., Ma, L., Wei, H., Zhang, J., He, X., et al. (2015). Coordinated regulation of arbuscular mycorrhizal fungi and soybean mapk pathway genes improved mycorrhizal soybean drought tolerance. Mol. Plant Microbe Interact. 28, 408-419. doi: 10.1094/MPMI-09-14-0251-R

Livak, K. J., and Schmittgen, T. D. (2001). Analysis of relative gene expression data using real-time quantitative PCR and the $2^{-\Delta \Delta C} \mathrm{~T}$ method. Methods 25, 402-408. doi: 10.1006/meth.2001.1262

Longmuir, K. J., and Johnston, J. M. (1980). Changes in CTP: phosphatidate cytidylyltransferase activity during rabbit lung development. Biochim. Biophys. Acta 620, 500-508. doi: 10.1016/0005-2760(80)90142-3

Mittler, R. (2006). Abiotic stress, the field environment and stress combination. Trends Plant Sci. 11, 15-19. doi: 10.1016/j.tplants.2005.11.002

Munns, R. (2005). Genes and salt tolerance: bringing them together. New Phytol. 167, 645-663. doi: 10.1111/j.1469-8137.2005.01487.x

Nielsen, T. H., Rung, J. H., and Villadsen, D. (2004). Fructose-2, 6-bisphosphate: a traffic signal in plant metabolism. Trends Plant Sci. 9, 556-563. doi: 10.1016/j.tplants.2004.09.004

Pastori, G. M., Mullineaux, P. M., and Foyer, C. H. (2000). Post-transcriptional regulation prevents accumulation of glutathione reductase protein and activity in the bundle sheath cells of maize. Plant Physiol. 122, 667-676. doi: 10.1104/pp.122.3.667

Peng, C., Wang, Y., Liu, F., Ren, Y., Zhou, K., Lv, J., et al. (2014). FLOURY ENDOSPERM6 encodes a CBM48 domain-containing protein involved in compound granule formation and starch synthesis in rice endosperm. Plant J. 77, 917-930. doi: 10.1111/tpj.12444

Péret, B., Swarup, K., Ferguson, A., Seth, M., Yang, Y., Dhondt, S., et al. (2012). AUX/LAX genes encode a family of auxin influx transporters that perform distinct functions during Arabidopsis development. Plant Cell 24, 2874-2885. doi: 10.1105/tpc.112.097766

Petrov, V., Hille, J., Mueller-Roeber, B., and Gechev, T. S. (2015). ROS-mediated abiotic stress-induced programmed cell death in plants. Front Plant Sci. 6:69. doi: 10.3389/fpls.2015.00069

Pilon-Smits, E. A., Terry, N., Sears, T., Kim, H., Zayed, A., Hwang, S., et al. (1998). Trehalose-producing transgenic tobacco plants show improved growth performance under drought stress. J. Plant Physiol. 152, 525-532. doi: 10.1016/S0176-1617(98)80273-3

Pinheiro, H. A., Silva, J. V., Endres, L., Ferreira, V. M., de Albuquerque Câmara, C., Cabral, F. F., et al. (2008). Leaf gas exchange, chloroplastic pigments and dry matter accumulation in castor bean (Ricinus communis L) seedlings subjected to salt stress conditions. Ind. Crops Prod. 27, 385-392. doi: 10.1016/j.indcrop.2007.10.003

Puranik, S., Jha, S., Srivastava, P. S., Sreenivasulu, N., and Prasad, M. (2011). Comparative transcriptome analysis of contrasting foxtail millet cultivars in response to short-term salinity stress. J. Plant Physiol. 168, 280-287. doi: 10.1016/j.jplph.2010.07.005

Redillas, M. C., Park, S. H., Lee, J. W., Kim, Y. S., Jeong, J. S., Jung, H., et al. (2012). Accumulation of trehalose increases soluble sugar contents in rice plants conferring tolerance to drought and salt stress. Plant Biotechnol. Rep. 6, 89-96. doi: 10.1007/s11816-011-0210-3

Remy, E., Cabrito, T. R., Baster, P., Batista, R. A., Teixeira, M. C., Friml, J., et al. (2013). A major facilitator superfamily transporter plays a dual role in polar auxin transport and drought stress tolerance in Arabidopsis. Plant Cell 25, 901-926. doi: 10.1105/tpc.113.110353

Seki, M., Ishida, J., Narusaka, M., Fujita, M., Nanjo, T., Umezawa, T., et al. (2002). Monitoring the expression pattern of around 7,000 Arabidopsis genes under ABA treatments using a full-length cDNA microarray. Funct. Integr. Genomics 2, 282-291. doi: 10.1007/s10142-002-0070-6

Shaar-Moshe, L., Hübner, S., and Peleg, Z. (2015). Identification of conserved drought-adaptive genes using a cross-species meta-analysis approach. BMC Plant Biol. 15:111. doi: 10.1186/s12870-015-0493-6

Shimobayashi, M., Oppliger, W., Moes, S., Jenö, P., and Hall, M. N. (2013). TORC1-regulated protein kinase Npr1 phosphorylates Orm to 
stimulate complex sphingolipid synthesis. Mol. Biol. Cell 24, 870-881. doi: 10.1091/mbc.E12-10-0753

Shu, L., Lou, Q., Ma, C., Ding, W., Zhou, J., Wu, J., et al. (2011). Genetic, proteomic and metabolic analysis of the regulation of energy storage in rice seedlings in response to drought. Proteomics 11, 4122-4138. doi: 10.1002/pmic.2010 00485

Song, C. P., Agarwal, M., Ohta, M., Guo, Y., Halfter, U., Wang, P., et al. (2005). Role of an Arabidopsis AP2/EREBP-type transcriptional repressor in abscisic acid and drought stress responses. Plant Cell 17, 2384-2396. doi: 10.1105/tpc.105.033043

Srinivasan, T., Kumar, K. R. R., Meur, G., and Kirti, P. (2009). Heterologous expression of Arabidopsis NPR1 (AtNPR1) enhances oxidative stress tolerance in transgenic tobacco plants. Biotechnol. Lett. 31, 1343-1351. doi: 10.1007/s10529-009-0022-5

Sudhakar, C., Lakshmi, A., and Giridarakumar, S. (2001). Changes in the antioxidant enzyme efficacy in two high yielding genotypes of mulberry (Morus alba L.) under $\mathrm{NaCl}$ salinity. Plant Sci. 161, 613-619. doi: 10.1016/S01689452(01)00450-2

Tarazona, S., García-Alcalde, F., Dopazo, J., Ferrer, A., and Conesa, A. (2011). Differential expression in RNA-seq: a matter of depth. Genome Res. 21, 2213-2223. doi: 10.1101/gr.124321.111

Toldi, O., Kovács, G., Kiss, E., Sorvari, S., and Scott, P. (2002). Altered fructose2, 6-bisphosphatase levels cause phenotypic changes and shift development in plants. Acta Biol. Szeged. 46, 15-16.

Wang, F. Z., Wang, Q. B., Kwon, S.-Y., Kwak, S. S., and Su, W. A. (2005). Enhanced drought tolerance of transgenic rice plants expressing a pea manganese superoxide dismutase. J. Plant Physiol. 162, 465-472. doi: 10.1016/j.jplph.2004.09.009

Witt, S., Galicia, L., Lisec, J., Cairns, J., Tiessen, A., Araus, J. L., et al. (2012). Metabolic and phenotypic responses of greenhouse-grown maize hybrids to experimentally controlled drought stress. Mol. Plant. 5, 401-417. doi: $10.1093 / \mathrm{mp} / \mathrm{ssr} 102$
Xiao, J., Li, H., Zhang, J., Chen, R., Zhang, Y., Ouyang, B., et al. (2006). Dissection of GA 20-oxidase members affecting tomato morphology by RNAi-mediated silencing. Plant Growth Regul. 50, 179-189. doi: 10.1007/s10725-006-9117-3

Xiong, L., Schumaker, K. S., and Zhu, J. K. (2002). Cell signaling during cold, drought, and salt stress. Plant Cell 14, 165-183. doi: 10.1105/tpc.000596

Xue, G.-P., Drenth, J., Glassop, D., Kooiker, M., and McIntyre, C. L. (2013). Dissecting the molecular basis of the contribution of source strength to high fructan accumulation in wheat. Plant Mol. Biol. 81, 71-92. doi: 10.1007/s11103012-9983-1

Xue, G.-P., McIntyre, C. L., Glassop, D., and Shorter, R. (2008). Use of expression analysis to dissect alterations in carbohydrate metabolism in wheat leaves during drought stress. Plant Mol. Biol. 67, 197-214. doi: 10.1007/s11103-0089311-y

Zhang, L., Liu, D. Y., and Shao, T. (2008). The feeding value and utilization prospect of Lolium multiflorum. Pratacult. Sci. 4, 132-136.

Zhao, H., Chen, J., Liu, J., and Han, B. (2015). Transcriptome analysis reveals the oxidative stress response in Saccharomyces cerevisiae. RSC Adv. 5, 22923-22934. doi: 10.1039/C4RA14600J

Zhu, J. K. (2001). Plant salt tolerance. Trends Plant Sci. 6, 66-71. doi: 10.1016/S1360-1385(00)01838-0

Conflict of Interest Statement: The authors declare that the research was conducted in the absence of any commercial or financial relationships that could be construed as a potential conflict of interest.

Copyright (c) 2016 Pan, Zhang, Wang, Ma, Zhou, Huang, Nie, Wang, Yang and Li. This is an open-access article distributed under the terms of the Creative Commons Attribution License (CC BY). The use, distribution or reproduction in other forums is permitted, provided the original author(s) or licensor are credited and that the original publication in this journal is cited, in accordance with accepted academic practice. No use, distribution or reproduction is permitted which does not comply with these terms. 